\title{
Reduced electron relaxation rate in multi-electron quantum dots
}

\author{
Andrea Bertoni, Massimo Rontani, Guido Goldoni, and Elisa Molinari \\ INFM National Research Center on nanoStructures and bioSystems at Surfaces (S3) and \\ Dipartimento di Fisica, Università degli Studi di Modena e Reggio Emilia, \\ Via Campi 213/A, 41100 Modena, Italy
}

(Dated: November 21, 2018)

\begin{abstract}
We use a configuration-interaction approach and Fermi golden rule to investigate electron-phonon interaction in realistic multi-electron quantum dots. Lifetimes are computed in the low-density, highly correlated regime. We report numerical evidence that electron-electron interaction generally leads to reduced decay rates of excited electronic states in weakly confined quantum dots, where carrier relaxation is dominated by the interaction with longitudinal acoustic phonons.

PACS numbers: 73.21.La, 73.22.Lp, 73.61.Ey, 72.10.Di
\end{abstract}


Semiconductor quantum dots (QDs) are artificially-confined systems where full control on the number of carriers and energy states can be achieved by either tuning the confinement potential or using external fields [1]. These confined systems can reach unprecedented regimes of large Coulomb-to-kinetic energy ratios, thus offering an ideal laboratory for the exploration of correlation effects at the nanoscale [2]. In addition, QD devices in the single-electron (SE) or multi-electron (ME) regime hold a primary role in new classes of applications, ranging from optoelectronics to biology and quantum information processing [3].

One fundamental question is how the discrete density of electronic states affects the relaxation time of excited electrons. The knowledge of carrier lifetime is obviously of great importance for many applications, and most significant for the implementation of solid-state gates for quantum information processing, which require a large number of elementary operations to be realized within the phase coherence time of the relevant degrees of freedom. It was early recognized that, since scattering from optical phonon is suppressed in weakly bound QDs (with confinement energy below $\sim 10 \mathrm{meV}$ in GaAs) [4, 5], the coupling with the longitudinal acoustic (LA) phonon continuum becomes the main source of scattering, thus limiting charge relaxation times to tens of nanoseconds [6]. Theoretical estimates of LA-phonon induced scattering rates were obtained in a SE approach and showed a satisfactory agreement with transport-spectroscopy experiments 7]. However, the technologically relevant problem of estimating the carrier lifetime when more than one electron are present in the device and, in general, the fundamental question of whether Coulomb interaction between carriers affects the relaxation of ME quantum states have received little attention [8].

In this Letter we report numerical evidence that carrier-carrier Coulomb interaction leads to reduced relaxation rates of excited ME states in weakly confined, strongly correlated QDs when the dominating intra-band scattering mechanism is the interaction with LA-phonons. The lifetime for a specific QD is shown to strongly depend on the number of carriers.

The calculation of ME states in QDs has been approached by several methods (for a review of quantum states in single and coupled QDs see Refs. 2 and 9). Since we are interested in the relaxation time of excited ME states, we need to know both ground and excited states with comparable accuracy. Our method of choice is the configuration interaction (CI) approach: the correlated wave functions of the ground and excited states are written as superpositions of Slater determinants (SDs), $\left|\Phi_{i}\right\rangle=\prod_{\alpha_{i}} c_{\alpha_{i}}^{\dagger}|0\rangle$, obtained by filling in the SE 
spin-orbitals $\alpha$ with the $N$ electrons in all possible ways $\left(c_{\alpha}^{\dagger}\right.$ creates an electron in level $\alpha$ ). The fully interacting Hamiltonian, written within the envelope function and effective mass approximations, is first block diagonalized, exploiting orbital and spin symmetries. Finally, the ME Hamiltonian is diagonalized in each symmetry sector, giving the full set of excited states of the system [10].

In order to evaluate quantitatively the relaxation time at zero temperature between given ME states $b$ and $a, \tau_{b \rightarrow a}$, we employ the Fermi golden rule between fully correlated states [11],

$$
\tau_{b \rightarrow a}^{-1}=\frac{2 \pi}{\hbar} \sum_{\boldsymbol{q}}\left|\sum_{i j} c_{b i}^{*} c_{a j}\left\langle\Phi_{i}\left|V_{\boldsymbol{q}}\right| \Phi_{j}\right\rangle\right|^{2} \delta\left(E_{b}-E_{a}-\hbar \omega_{\boldsymbol{q}}\right),
$$

where the ME states $\left|\Psi_{a}\right\rangle=\sum_{i} c_{a i}\left|\Phi_{i}\right\rangle$ have been written explicitly as linear combinations of SDs $\left|\Phi_{i}\right\rangle, V_{\boldsymbol{q}}$ is the interaction operator of an electron with a LA-phonon with wave vector $\boldsymbol{q}, E_{a}$ and $\hbar \omega_{\boldsymbol{q}}$ are the ME-state and phonon energies, respectively. We use the standard deformation-potential model for $V_{q}$ and a linear dispersion for the bulk LA-phonon branch [12, 13].

The SE levels are calculated for a cylindrical single or double QD, with confinement potential $V(\boldsymbol{r})=V_{z}(z)+\frac{1}{2} m^{*} \omega_{0}^{2}\left(x^{2}+y^{2}\right), V_{z}(z)$ representing the vertical confinement of a single or double quantum well, $\hbar \omega_{0}$ being the SE energy level spacing of a lateral twodimensional harmonic trap, and $m^{*}$ being the effective mass. We consider typical "vertical" GaAs/AlGaAs samples with effective cylindrical geometry [15, 16]. For this configuration, and neglecting spin-orbit coupling, the SE spin-orbitals can be given the separable form $\psi_{n m g \sigma}(\boldsymbol{r}, s)=\varphi_{n m}(x, y) \phi_{g}(z) \chi_{\sigma}(s)$, with $s=\uparrow, \downarrow$ the spin state, $n=0,1, \ldots$ the radial and $m=0, \pm 1, \pm 2, \ldots$ the angular quantum numbers related to the lateral confinement, and $g=0,1, \ldots$ labeling the eigenstate in the $z$ direction. Since the vertical confinement is much stronger than the lateral one, it is sufficient to consider $g=0$ for single QDs, and $g=0,1$ for QDs coupled via vertical tunneling. In the ME Hilbert space, the resulting global symmetries are the total orbital angular momentum $z$-component $M$, total parity $G$, total spin $S$, and its $z$-component $S_{z}$, which are used to block diagonalize the ME Hamiltonian. This numerical scheme has proved extremely reliable in reproducing the measured transport and inelastic light scattering spectra in single and coupled QDs [10, 24].

Equation (11) shows explicitly that the ME scattering rate $\tau_{b \rightarrow a}^{-1}$ can be thought of as a combination of SE scattering events, where a given configuration (i.e., a single SD) changes 
at most by the occupation of a single spin-orbital $\psi_{n m g \sigma}(\boldsymbol{r}, s)$. The coefficients $c_{a i}$ are determined by the solution of the interacting problem through the CI method. It should be noted that the SE matrix elements $\left\langle\Phi_{i}\left|V_{\boldsymbol{q}}\right| \Phi_{j}\right\rangle$ depend strongly both on the SE orbitals $\varphi_{n m}(x, y) \phi_{g}(z)$, possibly changing via confinement potential and external fields, and on the phonon wave vector $\boldsymbol{q}$. The magnitude of $\boldsymbol{q}$ is determined by the transition energy $E_{b}-E_{a}$, fixed, in turn, by the difference between the SE energies of initial and final levels plus the Coulomb energy. Indeed, typical LA-phonon wavelengths are comparable to the spatial extension of SE quantum states. Coulomb interaction, therefore, may affect $\tau_{b \rightarrow a}^{-1}$ through both the CI coefficients $c_{a i}$ and the SE matrix elements via shift of the transition energies $E_{b}-E_{a}$.

We start our discussion of ME-state relaxation times by considering the two-electron case. In Fig. 11we show the relaxation rate $\tau_{b \rightarrow a}^{-1}$ (solid curve) for the LA-phonon induced transition as a function of the confinement energy $\hbar \omega_{0}$ in a single $\mathrm{QD}$, where $b \equiv(M=1, S=0)$ is the correlated first excited state and $a \equiv(M=0, S=0)$ the ground state within the singlet sector. This transition is mostly composed of a SE $p \rightarrow s$ transition $[p \equiv(m=1, n=0)$, $s \equiv(m=0, n=0)$ ], with a "spectator" electron in level $s$ (see diagram in Fig. 11). For comparison, we show in Fig. 1 the corresponding non-interacting $p \rightarrow s$ relaxation rate (dashed curve) [6]. The relaxation rate of the correlated state is suppressed by a factor $\sim 2.5$ with respect to the SE transition. The two panels of the inset in Fig. 1 plot the same transition for the case of a double-dot sample with selected values of the inter-dot barrier. Here, the SE orbitals are coherently delocalized across the sample, with levels $(m n g)=(100)$ and $(000)$ corresponding to $p$ and $s$, respectively. The behavior is similar to the single QD system, with $\tau_{b \rightarrow a}^{-1}$ vanishing at the low and high energy sides, but oscillations appear at intermediate values of $\hbar \omega_{0}$; these oscillations, which increase in number with the width of the inter-dot barrier, are a pure SE effect and are due to the matching between the average separation of the two QDs and the phonon wavelength [14, 17, 18]. We stress that, in the ME case, even if $\tau_{b \rightarrow a}^{-1}$ is reduced by electron correlation with respect to the corresponding SE transition, it behaves qualitatively in the same way. Specifically, the values of the confinement energy and of external fields at which the scattering rate is suppressed (cf. Fig. 11) are the same.

Now consider the lowest-energy SE transition in a parabolic QD, in which case the SE gaps $\hbar \omega_{0}$ fix the energy of emitted (bulk) phonons $\hbar \omega_{\boldsymbol{q}}=\hbar \omega_{0}$. As the confinement energy 
changes, e.g., by tuning the gate potential, both the phonon energy and the SE orbitals vary. In the SE case the scattering rate variation is due to the combination of these two effects. For the two-electron case, however, $\hbar \omega_{\boldsymbol{q}}$ is in general different from $\hbar \omega_{0}$ due to Coulomb contribution. Therefore, in order to verify whether $\tau_{b \rightarrow a}^{-1}$ is reduced by a genuine correlation effect or simply by the change in the emitted phonon energy, we calculated $\tau_{b \rightarrow a}^{-1}$ by artificially fixing $\hbar \omega_{\boldsymbol{q}}$ at the SE gap $\hbar \omega_{0}$ also in the two-electron case. This is done by substituting the Dirac delta in Eq. (II) with $\delta\left(\hbar \omega_{0}-\hbar \omega_{\boldsymbol{q}}\right)$. We found that the scattering rate obtained in this way (triangles in Fig. 1) differs from the two-particle correlated case by only a few percents. Consequently, the reduction in $\tau_{b \rightarrow a}^{-1}$ is brought about by correlation only, since the transition energy and, therefore, the emitted phonon wave vector are only slightly affected by the interaction.

We show next that the reduction of the scattering rate brought about by Coulomb correlation is a more general effect and holds also for a larger number of electrons. In the CI approach, the ME transition rate between two specific states is the square of a sum of many SE transition amplitudes [Eq. (11)] and, due to interference effects among those amplitudes, there is no reason to believe a priori that the ME transition rates are larger or smaller than the SE ones. We found, however, that the calculated ME transition rate is, in general, smaller than the largest transition rate between two uncorrelated states of the QD, as discussed below.

In order to discuss the scattering rates for $N>2$, in Fig. 2 we show the LA-phonon induced scattering rate $\tau_{b \rightarrow a}^{-1}$ as a function of the emitted phonon energy $\hbar \omega_{\boldsymbol{q}}$ in QDs at selected lateral confinements and $2 \leq N \leq 6$. Each point in the graphs represents a transition between two correlated ME states. The first 20 eigenstates are considered, for each value of $N$; due to total spin conservation, only a few of all possible transitions are allowed. In each panel several ME transitions accumulate precisely at $\hbar \omega_{0}$, identified by downward arrows: these transitions correspond to the de-excitation of Kohn modes [1], i.e., the separable center-of-mass modes of the ME system, whose energies are exact multiples of $\hbar \omega_{0}$.

To compare the ME scattering rates with SE ones, we report in Fig. 2 (dashed curve) the scattering rate of the lowest $(p \rightarrow s)$ SE transition for a QD whose parabolic confinement energy matches the emitted-phonon energy, as implied by the SE picture. Note that, for such a curve, the graph abscissa indicates also the confinement energy. Since each ME 
transition has many SE components, in order to identify the transitions that are more directly comparable with the SE ones, we have indicated with the symbol $\mathbf{X}$ those processes whose highest-weight components of initial and final ME states can be mapped one into the other by destroying an electron occupying orbital $p$ (initial SD) and moving it to $s$ (final $\mathrm{SD})$ : the relaxation time of these specific ME transitions matches that of the $\mathrm{SE} p \rightarrow s$ relaxation once the correlation effects are neglected.

At the low-energy side of each plot we see that a few ME transitions have a scattering rate which is higher than the $\mathrm{SE}$ one, which seems to contradict results for $N=2$. However, note that here we are showing the ME scattering rates as a function of $\hbar \omega_{\boldsymbol{q}}$ at a given $\hbar \omega_{0}$, while in the SE case (dashed curve) the confinement energy is $\hbar \omega_{\boldsymbol{q}}$, as explained above. To single out the specific effects of Coulomb interaction on $\tau_{b \rightarrow a}^{-1}$, a more significant comparison is with SE transitions (solid curve) calculated with the lateral confinement energy $\hbar \omega_{0}$ used for the ME calculations (values indicated in Fig. 2), i.e. releasing the condition that equals the harmonic confinement to the phonon energy. In other words it is the solid curve of Fig. 2 that corresponds to the $p \rightarrow s$ SE transition contributing to the $i j$ summation of Eq. 1. As expected, the solid and dashed curves coincide at $\hbar \omega_{\boldsymbol{q}}=\hbar \omega_{0}$. For all considered cases, the scattering rate of ME transitions lies below the SE one with the same SE wave function and phonon energy; this is true, in particular, for Kohn modes.

The three QDs considered in Fig. 2 correspond to rather different Coulomb-to-kinetic energy ratios [19]: in terms of $\lambda=l_{0} / a_{\mathrm{B}}^{*}$, where $l_{0}=\sqrt{\hbar / m^{*} \omega_{0}}$ is the QD radius and $a_{\mathrm{B}}^{*}$ the effective Bohr radius, $\lambda=3.3,2.3,1.9$ for $\hbar \omega_{0}=1,2,3 \mathrm{meV}$ and GaAs parameters, respectively. The larger $\lambda$ (and the dot), the stronger the effect of correlation, keeping $N$ fixed [20]. Figure 2 shows that larger QDs with stronger correlation effects have lower scattering rates. The above discussion demonstrates that Coulomb interaction tends to decrease the scattering rate of ME states in weakly confined QDs. From the point of view of specific applications, if a low SE LA-phonon scattering rate is predicted for a specific sample and/or field configuration, then such desirable property will not be spoiled by Coulomb interactions.

The lifetime $\Gamma^{-1}$ of a given quantum state depends both on the scattering rates and on the number of channels of possible de-excitation, i.e., the density of states of symmetryallowed (spin-conserving) final states. In the ME system under study, an increasing number of particles leads to a larger number of states a given initial state can decay into. Such effect 
competes with the above prediction of smaller scattering rates. We report in Fig. 3 inset the inverse lifetime of the first 20 QD excited states for fixed $N$, considering different curves for $2 \leq N \leq 6$. First we note that, due to the varying density of states, the energy range covered by the transitions considered decreases as the number of electrons increases. The 20 decay times are reported, for each $N$, as a function of the difference between initial and final state energies, where the final state is the $N$-electron ground state. The points are connected with lines as a guide to the eye. Although the curves do not show a monotonic behavior, two global features emerge: (i) as the energy of the initial state increases, its lifetime lowers (ii) the lifetime of a quantum state increases with $N$, provided the excitation energy is fixed. Property (i) holds since the density of de-excitation channels increases with the initial state energy, while (ii) is due to the fact that scattering rates decrease as correlation effects become stronger. In the samples we considered, the effect (ii) is not obscured by (i). This is shown in Fig. 3. where the scattering time averaged over the first $20 \mathrm{ME}$ excited states is reported as a function of $N$ for three different QD samples.

To summarize, we quantitatively estimated the LA phonon-induced relaxation times of correlated ME excited states in weakly confined cylindrical GaAs/AlGaAs QDs. We find that electron-electron interaction contributes to reduce the scattering rate. Furthermore we showed that the correlated ME energy spectra mimic that of non-interacting QDs. In particular, the transition probability is suppressed for phonon energies whose wavelength matches the QD vertical dimension (when emission is inhibited also in the non-correlated case [14]), independently from the number of interacting electrons. We believe that these effects are of considerable relevance for the modeling of future nanodevices based on coherent electron dynamics and, in particular, for quantum computing proposals.

We acknowledge support from the Italian Ministry of Foreign Affairs (DGPCC), the Italian Ministry of Research under the FIRB (RBAU01ZEML) and COFIN (2003020984) programs, and INFM Iniziativa Trasversale Calcolo Parallelo 2005. We are grateful to F. Troiani for discussions.

[1] L. Jacak, P. Hawrylak, and A. Wójs, Quanum Dots (Springer, Berlin, 1998).

[2] S. M. Reimann and M. Manninen, Rev. Mod. Phys. 74, 1283 (2002). 
[3] F. Rossi, ed., Semiconductor Macroatoms: Basic Physics and Quantum-Device Applications (Imperial College Press, London, 2005).

[4] T. Inoshita and H. Sakaki, Phys. Rev. B 46, R7260 (1992).

[5] Due to the low lateral confinement, the electron-phonon coupling is weak in the samples considered. As a consequence we neglect the second-order process in which the emitted phonon is reabsorbed. For QDs in the strong coupling regime, where a full diagonalization of the Frölich Hamiltonian is in order to describe the polaron formation, see, for example, Refs. 21, 22, 23.

[6] U. Bockelmann, Phys. Rev. B 50, 17271 (1994).

[7] T. Fujisawa, D. G. Austing, Y. Tokura, Y. Hirayama, and S. Tarucha, Nature 419, 278 (2002).

[8] M. Brasken, S. Corni, M. Lindberg, J. Olsen, and D. Sundholm, Molecular Phys. 100, 911 (2002).

[9] M. Rontani, F. Troiani, U. Hohenester, and E. Molinari, Sol. Stat. Comm. 119, 309 (2001).

[10] M. Rontani, S. Amaha, K. Muraki, F. Manghi, E. Molinari, S. Tarucha, and D. G. Austing, Phys. Rev. B 69, 85327 (2004).

[11] Since we are considering an out-of-equilibrium process at $T \sim 0$, whose rate could be estimated by a transport pump-and-probe experiment like the one reported in Ref. 7, we neglect the second-order "in" scatterings.

[12] D. K. Ferry, Semiconductors (Macmillan, New York, 1991).

[13] Well converged results are obtained for the lowest $20 \mathrm{ME}$ states for $1 \leq N \leq 6$ using $56 \mathrm{SE}$ spin-orbitals, corresponding up to $\approx 3 \times 10^{5}$ SDs.

[14] A. Bertoni, M. Rontani, G. Goldoni, F. Troiani, and E. Molinari, Appl. Phys. Lett. 85, 4729 (2004).

[15] S. Tarucha, D. G. Austing, T. Honda, R. J. van der Hage, and L. P. Kouwenhoven, Phys. Rev. Lett 77, 3613 (1996).

[16] R. C. Ashoori, Nature 379, 413 (1996).

[17] P. Zanardi and F. Rossi, Phys. Rev. Lett. 81, 4752 (1998).

[18] A. Bertoni, M. Rontani, G. Goldoni, F. Troiani, and E. Molinari, Physica E 26, 427 (2005).

[19] R. Egger, W. Hausler, C. H. Mak, and H. Grabert, Phys. Rev. Lett. 82, 3320 (1999).

[20] M. Rontani and E. Molinari, cond-mat/0408454.

[21] S. Hameau, Y. Guldner, O. Verzelen, R. Ferreira, G. Bastard, J. Zeman, A. Lemaitre, and J. M. Gérard, Phys. Rev. Lett. 83, 4152 (1999). 
[22] A. Vagov, V. M. Axt, and T. Kuhn, Phys. Rev. B 67, 115338 (2003).

[23] U. Hohenester and G. Stadler, Phys. Rev. Lett. 92, 196801 (2004).

[24] C. P. García, V. Pellegrini, A. Pinczuk, M. Rontani, G. Goldoni, E. Molinari, B. S. Dennis, L. N. Pfeiffer, and K. W. West, submitted. 


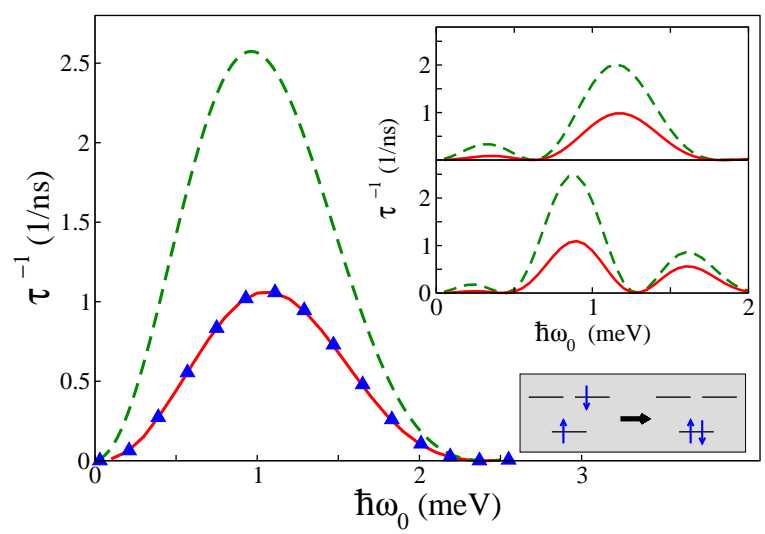

FIG. 1: Electron-LA phonon scattering rate at $\mathrm{T}=0$ for: dashed curve, $\mathrm{SE} p \rightarrow s$ transition, and, solid curve, two-electron $(M=1, S=0) \rightarrow(M=0, S=0)$ transition (see lower inset), for a cylindrical QD (GaAs layer $10 \mathrm{~nm}$ thick), as a function of the lateral confinement energy, $\hbar \omega_{0}$. Scattering rates for one and two electrons would be the same if correlation were switched off. Triangles show the two-electron rate calculated by artificially taking the energy of the emitted phonon equal to the confinement energy, as in the SE case: the values do not differ significantly from the "true" transition (solid line). The upper insets show the corresponding transition rates for a double dot with inter-dot barrier $3 \mathrm{~nm}$ (upper graph) and $8 \mathrm{~nm}$ (lower graph) wide, respectively. 


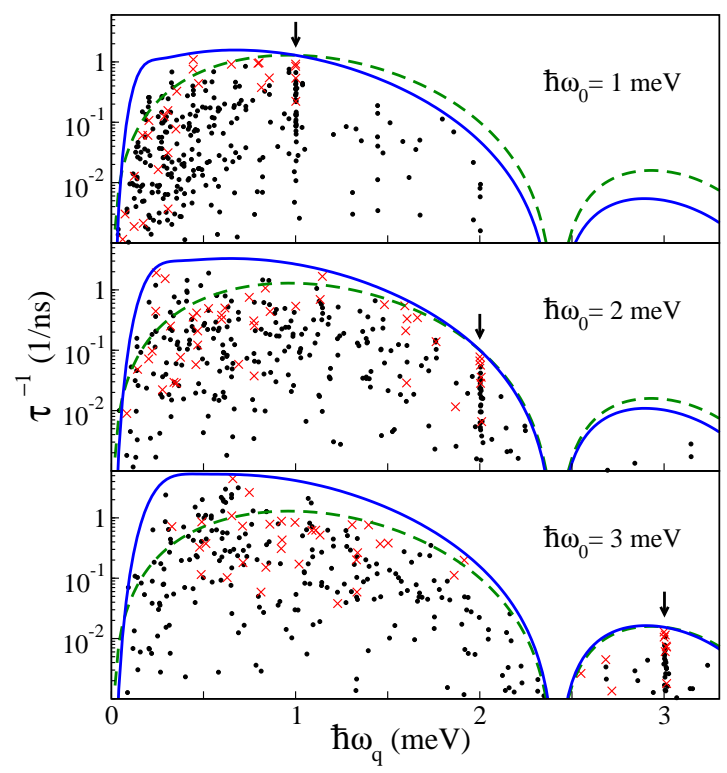

FIG. 2: Electron-LA phonon scattering rate, for three QDs with different lateral confinement energy $\hbar \omega_{0}$, as a function of the energy of the emitted phonon, $\hbar \omega_{\boldsymbol{q}}$. Dashed curves show the SE scattering rate of the lowest-energy transition $(p \rightarrow s)$. Solid curves refer to the same transition except orbitals are fixed to the value of $\hbar \omega_{0}$ indicated in figure. Dots and $\mathbf{X}$ symbols identify specific ME transitions from excited to ground states with $2 \leq N \leq 6$ (the first 20 excited states for each $N$ were considered), with the $\mathrm{X}$ symbols labeling ME transitions evolving into the non-interacting ones as correlation is switched off (see text). Arrows identify Kohn modes.

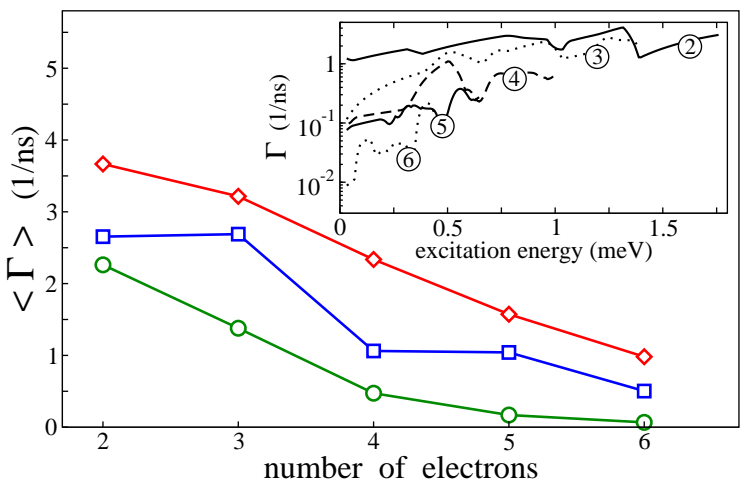

FIG. 3: Mean decay rate of correlated ME states with up to six electrons, due to electron-LA phonon scattering, for the three QDs of Fig. 2. with $\hbar \omega_{0}=1 \mathrm{meV}$ (circles), $2 \mathrm{meV}$ (squares), $3 \mathrm{meV}$ (diamonds). Inset: decay rate of the first 20 correlated ME states as a function of the excitation energy for the QD with $\hbar \omega_{0}=1 \mathrm{meV}$. The curves connecting the transitions considered are guides to the eye. The labels indicate the number of electrons. 